\title{
Emergency Medical Services and Remote Medical Oversight in Sequoia and Kings Canyon National Parks, $2011-2013$
}

\author{
Megann Young, MD; Thomas Kim, MD; Eli Lessard, MD; Danielle Campagne, MD; Susanne Spano, MD; \\ Leyla Farshidpour; Geoff Stroh, MD
}

From the UCSF Fresno Department of Emergency Medicine (Drs Young, Lessard, Campagne, Spano, Stroh, and Ms. Farshidpour); the UCSF Fresno Parkmedic Program (Drs Young and Stroh); the UCSF Fresno Wilderness Medicine Fellowship (Dr Spano); the Central California Emergency Medical Services Agency, Fresno, CA (Dr Stroh); and the University of Nevada Reno, Reno, NV (Dr Kim).

\begin{abstract}
Introduction-The University of California San Francisco Fresno Department of Emergency Medicine provides base hospital support for the Sequoia and Kings Canyon National Parks (SEKI) emergency medical services (EMS) system. This descriptive epidemiologic study reports reasons the park EMS system is used and interventions provided, detailing the nature of patient encounters, type and frequency of injuries and interventions, reasons for base hospital contact, and patient dispositions.

Methods-Patient charts for all EMS encounters in SEKI from 2011 to 2013 were included, and relevant data were extracted by a single reviewer.

Results-Of the 704 charts reviewed, $570(81 \%)$ were frontcountry patient encounters (within $1.6 \mathrm{~km}$ [1 mi] of a paved road); 100 (14\%) were backcountry; and 34 (5\%) occurred in undefined locations. Regarding sex and age, $58 \%$ of patients were men; $22 \%$ were younger than $18 \mathrm{y}$, and $15 \%$ were 65 y or older. More than $80 \%$ of calls occurred during the months of June through August. The most common complaints were extremity trauma (24\%), torso trauma (13\%), and lacerations (9\%). Almost $50 \%$ of patients were transferred to a higher level of care. Medications were administered to $37 \%$ of patients, with oxygen being the most common. Procedures were performed $49 \%$ of the time, primarily intravenous access and splinting. Base hospital contact was made $38 \%$ of the time, most commonly (54\%) for advice regarding disposition.

Conclusions - SEKI EMS providers encounter a wide variety of patients in various settings, including the backcountry. Resource allocation, training, and protocol development should be tailored to meet their needs.
\end{abstract}

Keywords: EMS, wilderness medicine, backcountry medicine, National Park Service

\section{Introduction}

The National Park Service (NPS) has unique needs in providing emergency medical services (EMS) because of diverse environments, remote locations, and unique patterns of illness and injury. ${ }^{1-4}$ Relatively few data have been published regarding this varied and challenging prehospital environment. This study describes the most common reasons why the NPS EMS system in

Corresponding author: Megann Young, MD, 155 N Fresno St, Fresno, CA 93701. Tel: 559-499-6440; e-mail: myoung @ fresno.ucsf.edu.

Submitted for publication October 2017.

Accepted for publication June 2018.
Sequoia and Kings Canyon National Parks (SEKI) is used. It also delineates the types of interventions provided, patient disposition, and the reasons why EMS providers seek online medical oversight via base hospital contact. By analyzing these data, we hope to provide data to EMS administrators and contribute to future planning and protocol development for the SEKI EMS system and other similar EMS systems.

Patient care in the NPS EMS system is primarily directed by offline medical oversight in the form of protocols written and approved by the NPS EMS advisory committee. Some parks also have online medical oversight provided by a local hospital and local EMS medical 
advisors. Although offline medical oversight forms the basis for most patient care, online medical oversight provides direct communication between physicians in the hospital and EMS providers in the field, allowing realtime recommendations regarding patient care. Online medical oversight can be particularly useful to NPS EMS providers because they may be required to provide patient care for hours or even days. This long duration of patient contact is relatively common within the NPS EMS system because many parks include remote locations with very long transport times to hospitals.

The NPS EMS providers are certified by the National Registry of Emergency Medical Technicians (NREMT) at the emergency medical responder, emergency medical technician (EMT), advanced EMT (AEMT), or paramedic level. Within the NPS, there is an additional EMS designation known as a parkmedic, which is obtained by a subset of NPS AEMTs. ${ }^{5,6}$ The scope of practice of the parkmedic is intermediate between that of an AEMT and a paramedic and includes advanced airway management (eg, supraglottic airway); needle thoracostomy; Taser dart removal; reduction of dislocations of the shoulders, patellae, and fingers or toes; administration of additional intravenous (IV) medications; and a selective spinal immobilization protocol that allows for decreased use of spinal motion restriction techniques. In SEKI specifically, the only credentialed NREMT levels are emergency medical responder, EMT, and AEMT. Parkmedics are credentialed by NREMT at the AEMT level, and SEKI does not use paramedics. Approximately 10 parkmedics work in SEKI each year. There are 3 ambulances within the park for frontcountry EMS response. In summer months a helicopter is available for use in backcountry rescues and is staffed by park EMS personnel. The NPS defines "frontcountry" as any location within $1.6 \mathrm{~km}(1 \mathrm{mi})$ of a paved road, whereas backcountry is any location more than a mile from a paved road. Responses to frontcountry calls are generally performed by ambulance or ranger patrol vehicle if the closest responder is an EMS-trained law enforcement ranger. In the backcountry, initial contact is often made by rangers stationed in the backcountry during the summer months; these rangers are exclusively trained at the EMT level and do not have any advanced life support (ALS) training or supplies. The ALS providers in SEKI are currently only stationed in the frontcountry and can respond to the backcountry by helicopter, hiking, or stock (horse or mule).

SEKI encompasses more than 350,000 hectares $(865,000$ acres) of mostly wilderness territory and is located approximately $80 \mathrm{~km}$ east of Fresno, CA. From 2011 to 2013, SEKI reported approximately 5 million visitors. $^{7}$ The University of California San Francisco, Fresno (UCSF Fresno), Department of Emergency
Medicine, in conjunction with Community Regional Medical Center (CRMC) in Fresno, California, serves as the local base hospital for SEKI. Online medical oversight is available at all times for SEKI EMS providers via the CRMC emergency department and is provided by physicians who are familiar with the NPS EMS scope of practice and patient care protocols. There are times when radio failure occurs and communication with the base hospital is not possible, and the EMS protocols include specific instructions for how to proceed in these cases. To maintain a reliable infrastructure for the provision of rapid medical care to individuals in need, more data are needed regarding the frequency and type of EMS calls. Although there is some information available regarding how many times EMS were activated, it is unclear for what reasons, what interventions were given, and with what outcomes. The last study of these elements for SEKI addressed patient encounters from 1986 to $1987 .{ }^{2}$ Johnson et $\mathrm{al}^{2}$ found that $77 \%$ of patients were treated and released; base hospital contact was made for $28 \%$ of all EMS calls; ALS care was provided to $10 \%$ of the patients; and basic life support (BLS) care was provided to $67 \%$ of all patients, with the remainder refusing treatment. More recent data are needed to ensure that the EMS system in SEKI is adequately staffed and equipped for the volume and type of calls received.

\section{Methods}

Patient charts for all EMS encounters in SEKI from January 1, 2011 through December 31, 2013 were included. NPS EMS providers enter all patient encounters into an electronic database, emsCharts. Deidentified patient charts were obtained from emsCharts and relevant data were extracted by a single reviewer into a password-protected spreadsheet in Excel (Microsoft, Redmond, WA). Data abstracted included date, age, sex, chief complaint, location of EMS call, mechanism of injury (for traumatic complaints), interventions performed by EMS providers, medications administered, disposition of patient, and whether contact was made with the base hospital.

Regarding patient disposition, there are 4 disposition possibilities: transfer by EMS, treat and release, transport by privately owned vehicle, and refusal of care. Transfer by EMS includes all patients transported by an EMS provider, whether by the NPS EMS ambulance or by another EMS provider (eg, helicopter, other local agency, litter carry, stock evacuation [use of a horse or mule to assist with patient transportation]). Patients may be transferred to several different hospitals or clinics, depending on location within the park and severity of injury, and the specific destination of each patient in this study is not known. The patients transported by EMS 
were all considered transfers to a higher level of care. There are 4 transfer-of-care possibilities: transport to a hospital, transport to a clinic, and rendezvous with either a helicopter or an ambulance from the local county EMS agency for transport to a hospital or clinic. Some patients, particularly in the backcountry, could be cared for by SEKI EMS providers and transferred directly to a helicopter or (in the frontcountry) a local ambulance provider without technically being transported (ie, moved) by SEKI EMS. These patients were also categorized as EMS transfers to a higher level of care. A disposition of "treated and released" indicates that some treatment was rendered, but the patient was deemed safe for discharge from EMS care without specific referral to a hospital or clinic for further treatment. A disposition of "privately owned vehicle" indicates that the patient was instructed to seek further care and treatment but that private transport would be acceptable, as opposed to ambulance or helicopter transport. Refusal of care is comparable to a patient leaving a hospital against medical advice and indicates that the patient declined medical evaluation or transport despite being advised to accept such care.

If base hospital contact was made, additional details were collected, including what questions were asked and what recommendations were made by the base hospital physician. For the purposes of this study, the reasons for making base hospital contact were defined as follows:

- Disposition advice: any advice regarding patient disposition. This could include questions surrounding appropriate mode of transport (eg, ALS vs BLS, ground vs helicopter vs private car); appropriate patient destination; and patient ability to sign out against medical advice.

- Patient counseling: any request by an EMS provider to have the base hospital physician speak directly to the patient.

- Management advice: any questions from the EMS provider regarding how to manage patient care, other than questions limited to medication advice.

- Medication advice/order: any request from an EMS provider for advice regarding what medications should be given, or what doses of medications should be given. This includes requests for medications that may be outside the particular protocol that the EMS provider is following or a request to give a patient his or her home medications.

- Unable to establish contact: any time an EMS provider attempted base hospital contact but was unable to establish communication.

The data were analyzed using descriptive statistics. Data are presented as percentages or as mean \pm SD with range, as appropriate. Approval was obtained from the institutional review board at Community Regional Medical Center.

\section{Results}

All EMS patient encounters in SEKI over the study period were reviewed, totaling 704 charts. Of these, 296 (42\%) were female, $406(58 \%)$ were male, and $2(<1 \%)$ did not include a documented sex. Most were aged 18 to $64 \mathrm{y}(\mathrm{n}=443 ; 63 \%) ; 22 \%(\mathrm{n}=152)$ were $17 \mathrm{y}$ or younger, and $15 \%(\mathrm{n}=105)$ were $65 \mathrm{y}$ or older. Four charts $(<1 \%)$ did not include a documented age. More than $80 \%(n=568)$ of all EMS calls occurred during the summer months of June through August (Figure 1). The majority of patient encounters $(n=570 ; 81 \%)$ occurred in the frontcountry. Only 100 encounters (14\%) occurred in the backcountry, or more than 1 mile from a paved road. For 34 encounters (5\%) the location was undocumented or unclear. The EMS providers made contact with base hospital physicians 268 times (38\%) during the study period.

Chief complaint as documented in the chart was available for all patients (Figure 2). The most common complaints were extremity trauma $(n=166 ; 24 \%)$, torso trauma $(n=92 ; 13 \%)$, and lacerations $(n=63 ; 9 \%)$. These traumatic injuries were followed in frequency by a number of medical complaints, especially shortness of breath $(\mathrm{n}=50 ; 7 \%)$ and dizziness $(\mathrm{n}=42 ; 6 \%)$. Insect bites were also a common chief complaint, representing 5\% $(n=35)$ of all patient encounters. Overall, there were 15 EMS calls per 100,000 visitors during the 3-y study period. Among these, EMS calls for traumatic injuries occurred at a rate of 7 per 100,000 visitors, whereas EMS calls for medical complaints occurred at a rate of 6 per 100,000 visitors. Calls categorized as other than medical or trauma occurred in 2 out of 100,000 visitors.

Medications were administered in 263 encounters (37\%), with oxygen being the most common $(n=197 ; 28 \%)$,

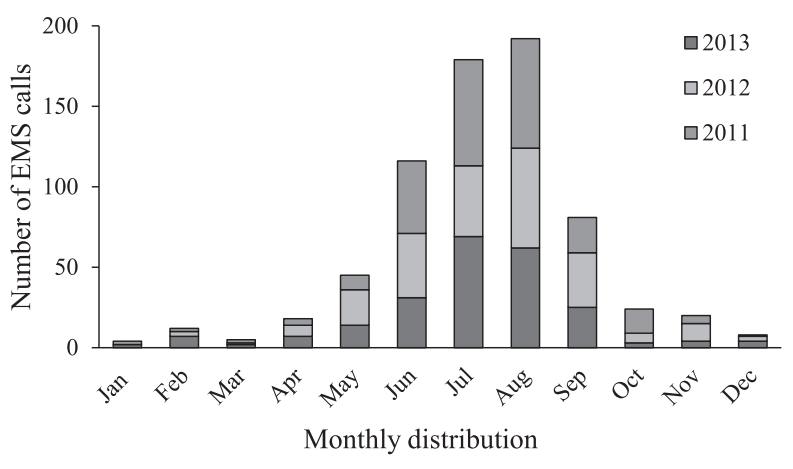

Figure 1. Monthly distribution of emergency medical services (EMS) cases in Sequoia and Kings Canyon National Parks over 3 y $(n=704)$. 


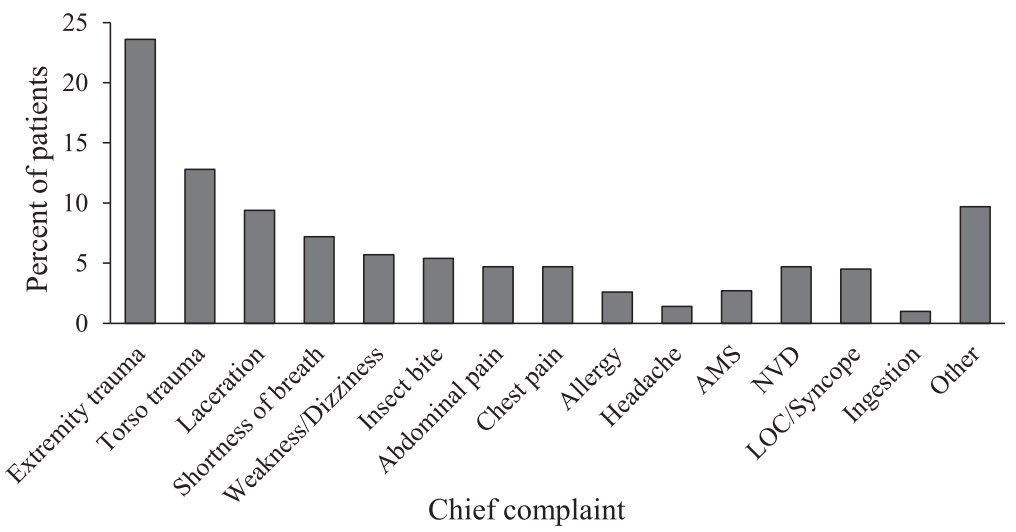

Figure 2. Chief complaint by percent for all patients (n=704). AMS, altered mental status; NVD, nausea/vomiting/diarrhea; LOC, loss of consciousness.

followed by ondansetron $(\mathrm{n}=49 ; 7 \%)$ and IV normal saline $(\mathrm{n}=35 ; 5 \%)$. Other medications were used relatively infrequently (Figure 3).

Although vital signs and a physical examination were documented in all but 5 cases, ALS providers performed further interventions in 292 encounters (41\%), most commonly IV access $(n=129 ; 18 \%)$ and splinting or other immobilization $(\mathrm{n}=127 ; 18 \%)$. Airway maneuvers $(\mathrm{n}=6$; $0.8 \%)$ and advanced cardiac life support $(\mathrm{n}=1 ; 0.1 \%)$ were performed infrequently (Figure 4). The patient disposition was available in all but 5 cases and categorized in 1 of 4 ways: transfer by EMS ( $\mathrm{n}=324 ; 46 \%)$, transfer by privately owned vehicle $(\mathrm{n}=129 ; 18 \%)$, treated and released $(\mathrm{n}=128$; $(18 \%)$, or refused care $(\mathrm{n}=113 ; 16 \%)$ (Figure 5).

\section{BASE HOSPITAL SUBGROUP}

The 268 encounters for which base hospital contact were made were analyzed as a subgroup. There were 93 such calls in 2011 (39\% of all EMS calls for that year), 103 in 2012 (44\% of all EMS calls), and 72 in 2013 (31\% of all EMS calls). They were categorized based on chief complaint (Figure 6) and reason for call-in (Figure 7). Although extremity trauma remained the most common chief complaint in this group, the frequency dropped to $18 \%(\mathrm{n}=128)$, and torso trauma fell to $10 \%(\mathrm{n}=71)$. "Other" complaints that did not fit a category were documented $13 \%$ of the time ( $n=92)$. A number of medical complaints, such as shortness of breath, weakness, and abdominal pain, were noted 7 to $8 \%$ of the time, as were insect bites. In this group lacerations represented only $5 \%$ of chief complaints $(n=35)$. The most common reason for initiation of base hospital contact was disposition advice $(\mathrm{n}=177 ; 66 \%)$. Medication advice or order was requested in $11 \%$ of calls $(n=30)$, patient counseling was requested in $6 \%(\mathrm{n}=16)$, and management advice was requested in $15 \%(n=39)$ of calls. For $8 \%$ of calls $(n=21)$, the reason for contacting the base hospital was

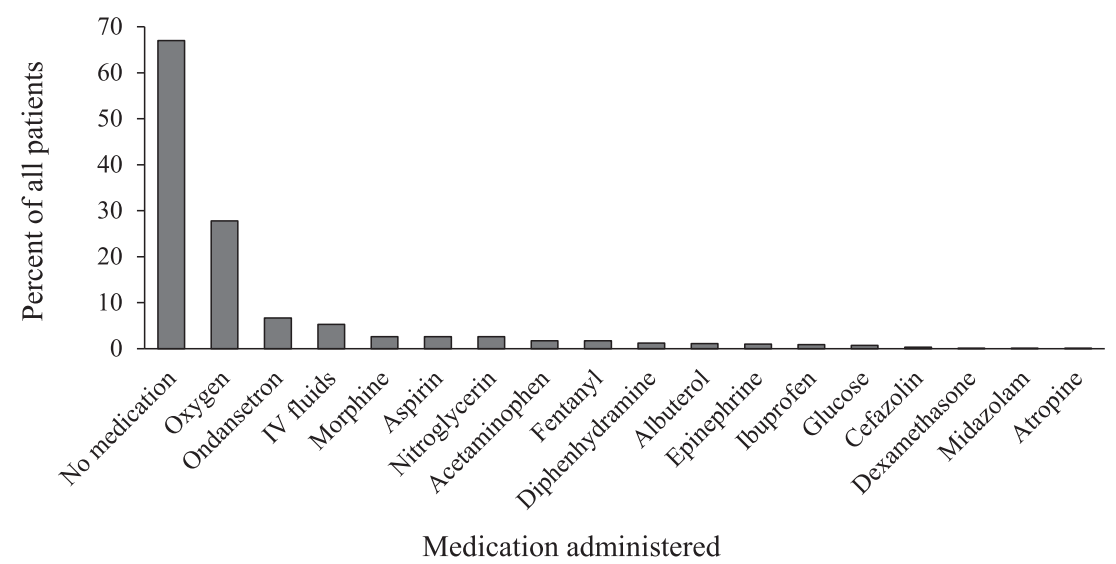

Figure 3. Medication administration by percent of all patients $(n=704)$. Percentages total more than $100 \%$ because some patients received multiple medications. IV, intravenous. 


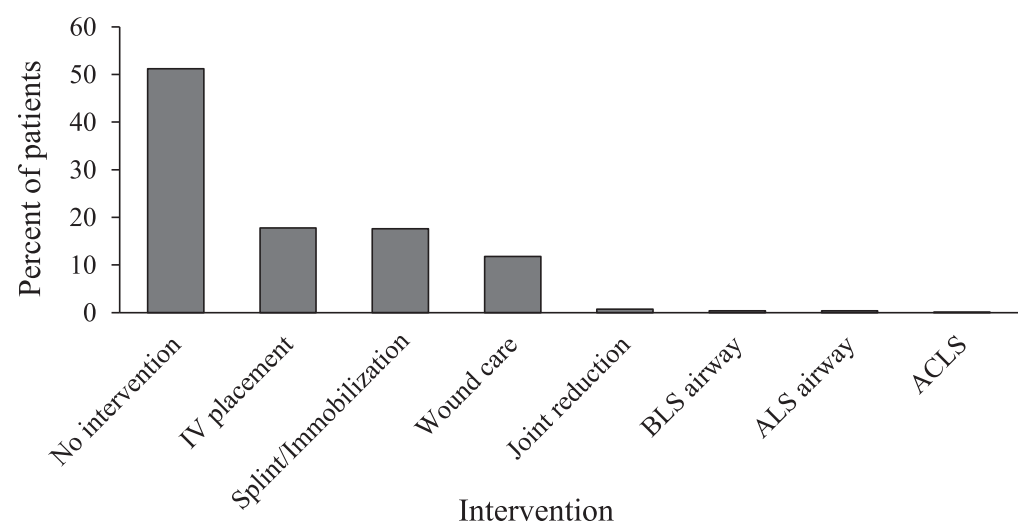

Figure 4. Emergency medical services provider intervention by percentage for all patients ( $n=704)$. Percentages total more than $100 \%$ because some patients received multiple interventions. ACLS, advanced cardiac life support; ALS, advanced life support; BLS, basic life support; IV, intravenous.

unclear. In $14 \%$ of calls $(n=38)$, questions were asked that encompassed more than one category (disposition advice plus medication advice $(\mathrm{n}=17 ; 6 \%)$; disposition advice plus management advice $(\mathrm{n}=12 ; 4 \%)$; disposition advice plus patient counseling $(\mathrm{n}=5 ; 2 \%)$; and management advice plus medication order $(\mathrm{n}=4 ; 1 \%)$. In only one case was contact attempted but not made; reasons for failure of contact are unclear.

\section{BACKCOUNTRY SUBGROUP}

The 100 encounters that occurred in the backcountry were also analyzed as a subgroup and compared with the frontcountry cohort. Nearly one quarter of patients in the backcountry cohort were female $(23 \% ; n=23)$, compared with $45 \%$ in the frontcountry cohort $(n=257)$. The average age in each group was similar, $46 \pm 16(7-76)$ y for the backcountry cohort and $43 \pm 23(2$ mo-90 y) years for the frontcountry cohort. However, among the backcountry cohort, $5 \%$ of patients $(n=5)$ were younger than 18 y and
$8 \%(\mathrm{n}=8)$ were $65 \mathrm{y}$ or older. In comparison, among the frontcountry cohort, $24 \%$ of patients $(n=140)$ were younger than $18 \mathrm{y}$ and $16 \%(\mathrm{n}=94)$ were $65 \mathrm{y}$ or older.

Three groups (total patients, frontcountry group, and backcountry group) were each categorized by chief complaint (Figure 8), medications administered (Figure 9), and patient disposition (Figure 10). Some notable differences were found in the backcountry group with regard to chief complaint. Although extremity trauma remained the most common, with similar frequency to the group at large $(\mathrm{n}=24 ; 24 \%)$, it was followed by medical complaints such as nausea and vomiting $(\mathrm{n}=14 ; 14 \%)$ and abdominal pain $(n=8 ; 8 \%)$. "Other" complaints represented $15 \%$ $(n=15)$ of all backcountry encounters, whereas torso trauma was noted in only $7 \%$ of cases $(n=7)$ and lacerations in only $4 \%(n=4)$. Complaints of insect bites and loss of consciousness were absent in this group, with no occurrences. Oxygen $(\mathrm{n}=28 ; 28 \%)$ and ondansetron $(\mathrm{n}=13 ; 13 \%)$ remained the most commonly administered medications. However, pain medications such as morphine

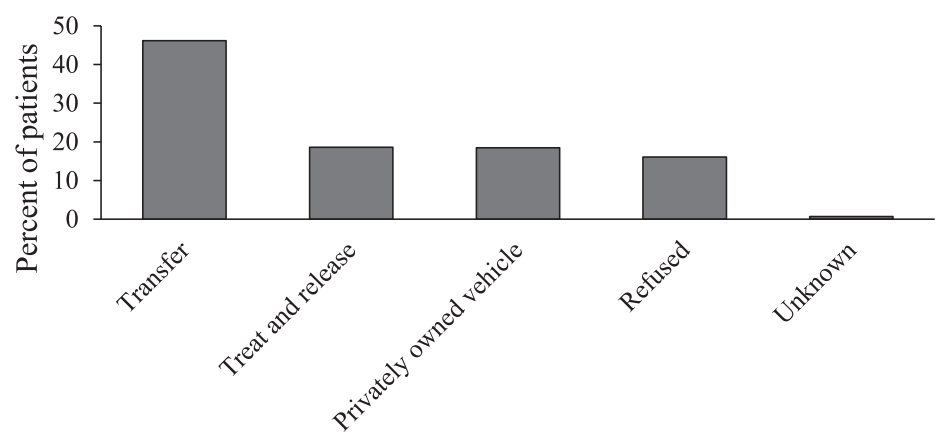

Disposition

Figure 5. Patient disposition by percentage of all patients $(n=704)$. 


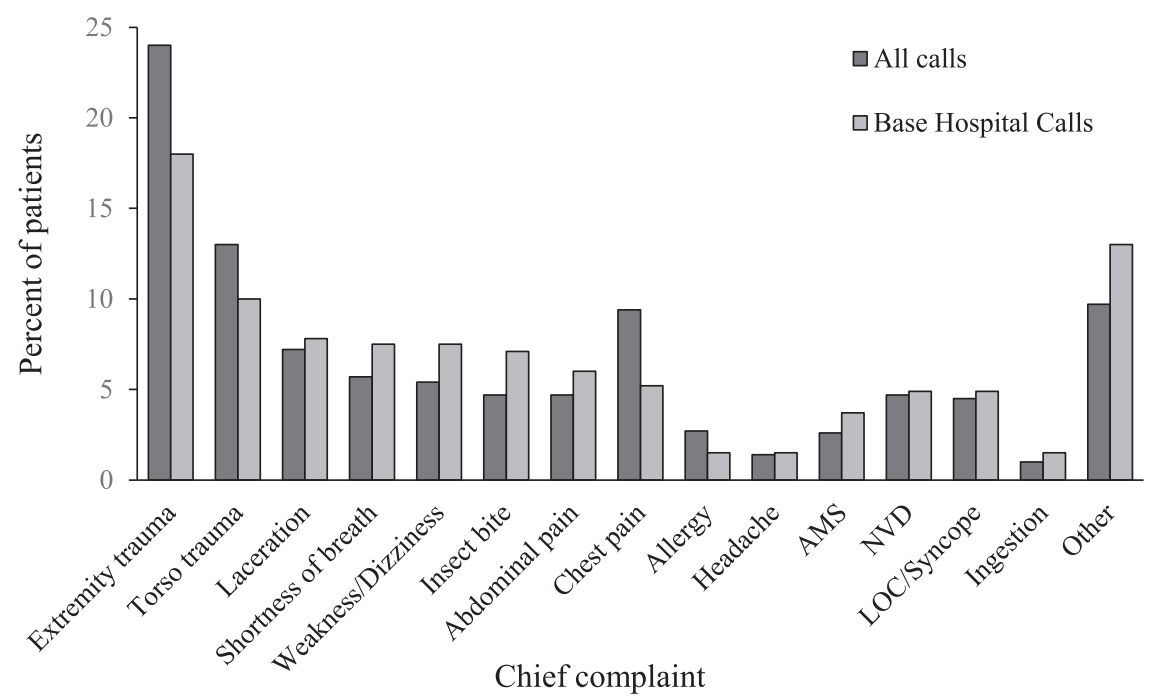

Figure 6. Chief complaint in base hospital subgroup ( $n=268)$ and entire study group ( $n=704)$, by percentage. AMS, altered mental status; LOC, loss of consciousness; NVD, nausea/vomiting/diarrhea.

$(\mathrm{n}=8 ; 8 \%)$ and ibuprofen $(\mathrm{n}=5 ; 5 \%)$ were administered more often in the backcountry cohort, as were IV fluids $(\mathrm{n}=7 ; 7 \%)$. Cefazolin $(\mathrm{n}=2 ; 2 \%)$ and atropine $(\mathrm{n}=1 ; 1 \%)$ were only administered in the backcountry setting. On the other hand, albuterol $(\mathrm{n}=8 ; 1 \%)$, dexamethasone $(\mathrm{n}=1$; $0.2 \%)$, and midazolam $(\mathrm{n}=1 ; 0.2 \%)$ were only administered in the frontcountry. Much lower rates of treat and release $(n=9 ; 9 \%)$ were identified in the backcountry cohort, with small increases in other dispositions.

\section{Discussion}

This retrospective epidemiologic study identified trends that will be useful in training and further protocol development for SEKI parkmedics, backcountry and wilderness EMS providers, or other EMS providers in austere settings.

We identified 2 general trends. First, the greatest number of calls were received in the summer months

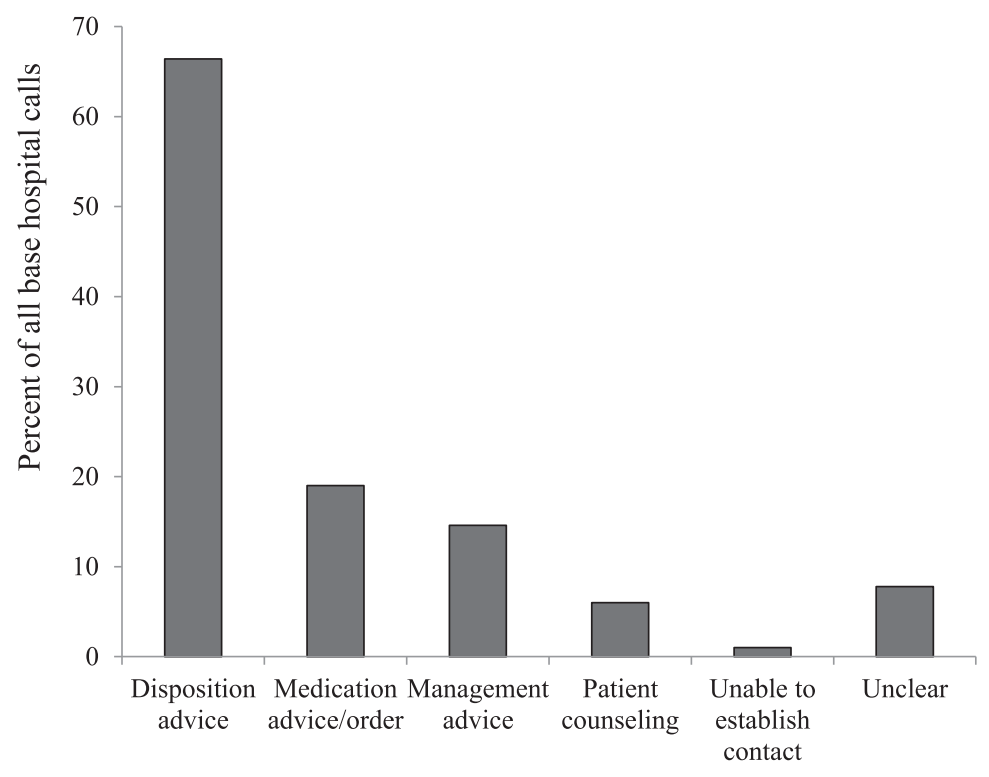

Type of base hospital advice requested

Figure 7. Reason for contacting base hospital $(\mathrm{n}=268)$, by percentage. Percentages total more than $100 \%$ because some calls requested multiple types of advice. 


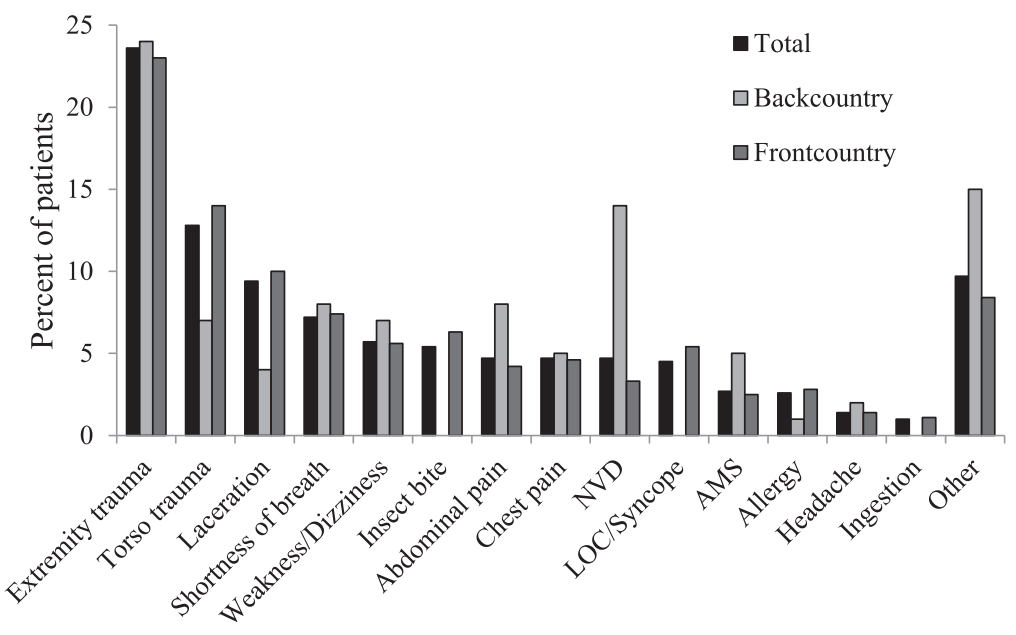

Chief complaint

Figure 8. Chief complaint for backcountry $(n=100)$ and frontcountry $(n=570)$ subgroups and entire study group ( $n=704)$, by percentage. AMS, altered mental status; LOC, loss of consciousness; NVD, nausea/vomiting/diarrhea.

of June, July, and August. These data are consistent with the NPS's visitor use statistics indicating that the greatest influx of visitors in SEKI occurs during these same months. ${ }^{7}$ Second, there were more requests for EMS assistance in frontcountry areas than backcountry areas. This result was expected because frontcountry regions in SEKI are more accessible and therefore draw greater numbers of visitors. The frontcountry, because of ease of access, is more likely to see visitors at extremes of age. We assumed that backcountry travel was more likely to be undertaken by younger, more seasoned hikers because of the more rugged terrain and longer hiking periods. Our data support this assumption, indicating relatively few patients at the extremes of age in the backcountry cohort. Lastly, it has been suggested in previous research that availability of cellular phone signal may affect the rates of EMS calls. ${ }^{1}$ In SEKI,

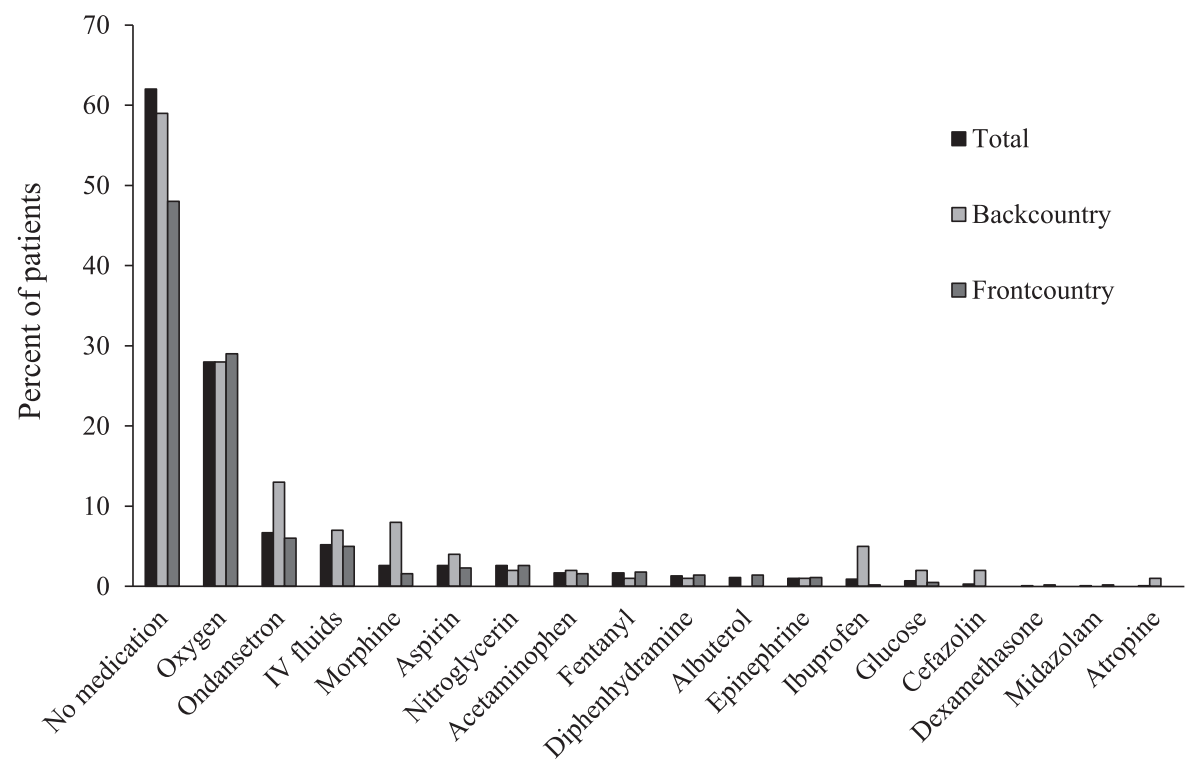

Medications administered

Figure 9. Medications administered for backcountry $(n=100)$ and frontcountry $(n=570)$ subgroups, as well as entire study group $(n=704)$, by percentage. IV, intravenous. 


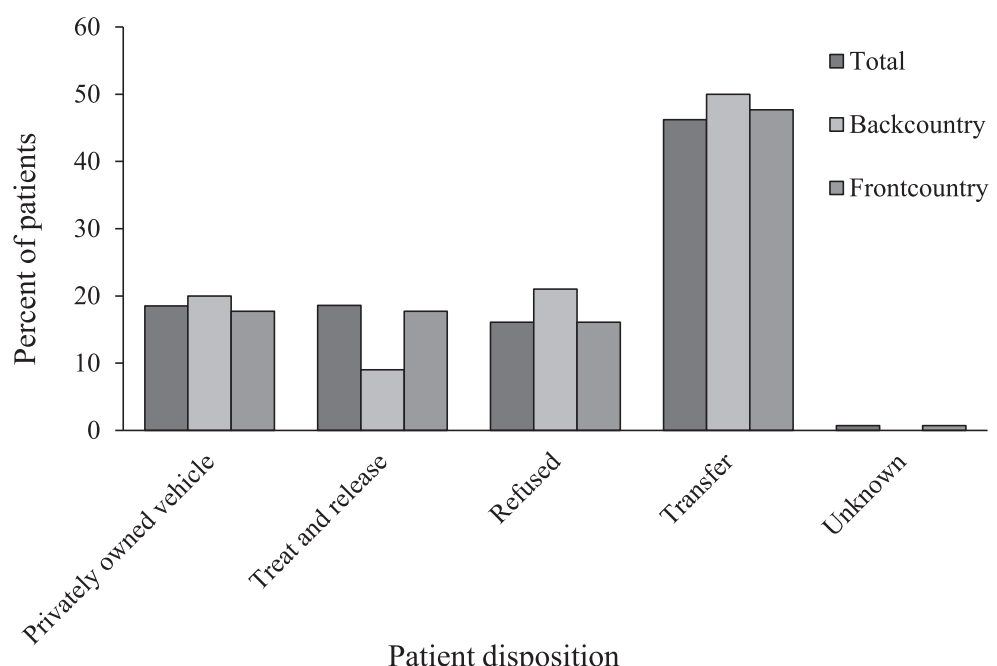

Figure 10. Patient disposition for backcountry $(n=100)$ and frontcountry $(n=570)$ subgroups as well as entire study group $(n=704)$, by percentage.

cellular phone reception is available in certain frontcountry areas, but it is largely unavailable in the backcountry areas. It seems likely that this contributes to the greater use of the EMS system in frontcountry areas.

Our data suggest that SEKI visitors request EMS assistance in roughly equal numbers for chief complaints regarding medical issues and trauma. These findings are consistent with a study conducted in 2015 reporting that the incidence of trauma and medical calls are roughly equal within the national park system. $^{3}$

\section{BASE HOSPITAL CONTACT}

We were interested in the incidence of and reasons for EMS contact with the base hospital at CRMC. As one of relatively few parks in the NPS with online medical oversight, SEKI benefits from real-time physician support of prehospital patient care. Only once in the $3 \mathrm{y}$ of the study was base hospital contact unsuccessful, suggesting the reliability of this model. It is not surprising that the most common questions for the base hospital physician were related to disposition and medication advice. Given the remote nature of the NPS, dispositions necessitating follow-up in an acute care setting (transfer, privately owned vehicle) represent significant resource commitment to the SEKI EMS system and inconvenience to the patient and accompanying individuals. Therefore, frequent basehospital contact to confirm disposition is to be expected. Moreover, it is notoriously difficult to create protocols to assist with disposition, particularly in an EMS system as varied in environment and topography as the NPS. This necessarily would lead to frequent call-ins for disposition advice. As far as medication advice is concerned, because transport times may exceed $2 \mathrm{~h}$ from even some frontcountry areas, EMS providers may need additional medication orders beyond what is specified in their standing (offline) protocols. Proportionally more calls were placed for medical complaints (54\%) vs trauma (33\%), perhaps indicating diagnostic uncertainty or potential complexity of care issues.

The incidence of base hospital contact increased from $28 \%$ in 1986 to 1987 to $38 \%$ in our study. ${ }^{2}$ This may be partly attributable to changes in mandatory call-in criteria delineated in NPS EMS protocols as well as advances in technology; however, the relatively large percentage of patient contacts resulting in base hospital calls suggests that prehospital providers often simply have questions regarding either medical care issues or difficult disposition situations with some potential medicolegal risk. We believe this result supports online medical oversight availability whenever and wherever possible. With modern communication improving and becoming more affordable, this is possible throughout the vast majority of the NPS, even in the backcountry. Although the study by Johnson et $\mathrm{al}^{2}$ reports data by EMS provider level (ALS vs BLS), this information was unavailable for our data set. Another notable difference between the 1986-1987 study and our study is the number of EMS calls; whereas Johnson et $\mathrm{al}^{2}$ report 434 patient contacts in 1 y, we found 235 \pm 5 (range, 231-241) EMS calls per year during our 3-y study period. It is not immediately clear why EMS calls decreased while visitation increased. ${ }^{7}$ The availability of cellular phones and devices such as personal locator beacons presumably makes EMS contact easier than previously, which 
would tend to increase the number of calls. Further study is required to investigate possible explanations for this finding.

\section{FRONTCOUNTRY VS BACKCOUNTRY}

Of particular interest were different incidences of patient complaints in the backcountry compared with the frontcountry. Only 35 backcountry hikers $(35 \%)$ contacted EMS for traumatic injuries (defined as extremity trauma, torso trauma, and lacerations), whereas 268 frontcountry visitors $(47 \%)$ contacted EMS for traumatic injuries. Proximity to EMS and ability to contact dispatch may have more to do with these differences than other factors. However, this could also be due to better preparation by backcountry hikers because they are typically more experienced and plan for the care of minor traumatic injuries. Individuals in the backcountry were more likely to request EMS assistance for medical issues such as nausea, vomiting, diarrhea, and abdominal pain. This may be because the sequelae of illnesses such as gastroenteritis may not be as worrying for individuals in the frontcountry, who have adequate access to water, sanitation facilities, and transportation. Backcountry hikers may not be as well equipped to deal with challenges posed by dehydration and poor sanitation, largely because of a lack of available potable water and restroom facilities, thus triggering a call to EMS.

Differences were also identified in medication administration between frontcountry and backcountry regions. In the backcountry, proportionally more ondansetron, IV fluids, morphine, and cefazolin were administered. We attribute the increase in morphine administration to longer transportation times for backcountry patients, hence the need for longer-lasting pain management. Increased administration of ondansetron and IV fluids are likely because of the greater number of backcountry cases of dehydration related to higher altitudes and limited water supply. Elevations within SEKI range from $417 \mathrm{~m}$ (1370 $\mathrm{ft})$ up to $4418 \mathrm{~m}(14,494 \mathrm{ft})$, with the majority of the higher elevations in the backcountry areas. Frontcountry areas go to roughly $2255 \mathrm{~m}(7400 \mathrm{ft})$, whereas the highest point in the backcountry is the peak of Mt Whitney at $4418 \mathrm{~m}(14,494 \mathrm{ft}){ }^{8}$ Cefazolin is typically only administered for open fractures and is a time-dependent therapy in our NPS protocols. It would be rare for an EMS provider in the frontcountry to be on scene with a patient for a prolonged period and also rare for a frontcountry patient to be more than a few hours from a hospital that could provide definitive care. These factors combine to make cefazolin administration less likely in the frontcountry than in the backcountry.
In the frontcountry, nitroglycerin, fentanyl, and diphenhydramine were administered more often. Diphenhydramine is used in cases of allergic reactions and insect bites, chief complaints that were not as common in the backcountry. Albuterol was only administered in the frontcountry. We surmise that some of this difference is due to the fact that backcountry hikers who are known to have asthma will typically carry their albuterol inhalers. Most years, including the years of this study, the backcountry ranger stations in SEKI are staffed by rangers trained at the EMT level. This level of training does not allow administration of albuterol unless the patient has his or her own, which would tend to decrease the likelihood of albuterol administration in the backcountry setting.

There were also differences between disposition in frontcountry and backcountry settings. A greater percentage of individuals in the frontcountry $(n=103 ; 18 \%)$ were treated and released compared with the backcountry $(\mathrm{n}=9 ; 9 \%)$. This difference is consistent with the idea that backcountry hikers are often much farther from alternate transport options, thus making EMS transport the more viable option.

\section{PATIENT DISPOSITION}

The most common method of disposition was transport by EMS $(n=324,46 \%)$. This represents an increase from the earlier study in which only $25 \%$ of patients were transported by the EMS system. ${ }^{2}$ We do not have a definitive explanation for this change, though we can postulate that there has been an improvement in resource availability over the last few decades, making EMS transport realistic in a higher proportion of cases. It is also possible that the changing medicolegal landscape has led to more conservative dispositions. It must be considered that the increased number of base hospital call-ins has contributed to this finding as well.

\section{LIMITATIONS}

Because of the retrospective nature of our study, there are some recognized limitations. Data collected were limited to those available through EMS documentation and cannot account for inaccurate documentation or incidents not documented by EMS. However, SEKI has an aggressive quality assurance process with $100 \%$ of all charts being reviewed by a physician. The authors' experience with the prehospital charting is that it is generally thorough and complete. A further limitation is the relatively small number of EMS calls. SEKI typically has approximately 250 EMS calls per year, which are handled by a staff of 10 to 12 ALS providers and 30 to 50 BLS providers, depending on the year and season. The number of cases in a 3-y study is only 704 . However, the patient contact time is usually quite long - typically 2 to $4 \mathrm{~h}$ and up to days in the backcountry-leading to a very 
different EMS provider experience. There is often time for multiple assessments and more thorough documentation than in an urban system, and thus we feel that our data are likely to be accurate. Lastly, although our system differs from urban EMS, the patient contact and transport issues are relevant to any rural EMS system covering sparsely populated areas or with limited EMS resources.

\section{Conclusions}

Although the majority of EMS activity occurred in accessible areas, the number of remote encounters supports the continued investment in backcountry EMS in SEKI and other similar parks. High volumes in the summer months, with a high percentage of traumatic injuries, support a robust summer EMS presence and continued education on trauma management. Although many drugs were rarely, if ever, administered, and advanced cardiac life support/airway procedures were uncommon, the potential for lifesaving interventions likely precludes any significant restructuring of the parkmedic tool kit. The use of dispositions such as treat and release and privately owned vehicle was common. Base hospital support was used primarily for disposition advice, leaving little room for decreasing base hospital use through protocol development. We feel that the results of this study support the current structure and function of the SEKI EMS system, particularly the consistent availability of online medical oversight.

Author Contributions: Study concept and design (TK, EL, MY, SS); obtaining funding (not applicable); acquisition of the data (LF); analysis of the data (EL, MY); drafting of the manuscript (TK, EL, MY, SS, LF); critical revision of the manuscript (TK, EL, MY, DC, GS); approval of final manuscript (DC, MY).
Financial/Material Support: None.

Disclosures: UCSF Fresno provides medical oversight for SEKI, and several faculties have paid positions with the National Park Service.

\section{References}

1. Smith WR. Integration of tactical EMS in the National Park Service. Wilderness Environ Med. 2017;28(2S): S146-3.

2. Johnson J, Maertins M, Shalit M, Bierbaum TJ, Goldman DE, Lowe RA. Wilderness emergency medical services: the experiences at Sequoia and Kings Canyon National Parks. Am J Emerg Med. 1991;9(3):211-6.

3. Lane JP, Taylor B, Smith WR, Wheeler AR. Emergency medical service in the US National Park Service: a characterization and two-year review, 2012-2013. Wilderness Environ Med. 2015;26(4):531-5.

4. Declerck MP, Atterton LM, Seibert T, Cushing TA. A review of emergency medical services events in US national park from 2007 to 2011. Wilderness Environ Med. 2013;24 (3):195-202.

5. Kaufman TI, Knopp R, Webster T. The Parkmedic Program: prehospital care in the national parks. Ann Emerg Med. 1981;10(3):156-60

6. Spano SJ. Chapter 116: National Park Service medicine. In: Auerbach P, ed. Auerbach's Wilderness Medicine. 7th ed. Philadelphia, PA: Elsevier; 2017:2487-97.

7. NPS Stats: National Park Service visitor use statistics. Available at: https://irma.nps.gov/Stats. Accessed June 19, 2017.

8. Sequoia \& Kings Canyon: driving mountain roads. Available at: https://www.nps.gov/seki/planyourvisit/drivingmountain-roads.htm. Accessed April 29, 2018. 